\title{
Monte Carlo Simulation of Particle Radiation in High Altitude Solid Rocket Plumes
}

\author{
Jonathan M. Burt ${ }^{1}$ and Iain D. Boyd ${ }^{2}$ \\ Department of Aerospace Engineering \\ University of Michigan, Ann Arbor, MI 48109
}

\begin{abstract}
Several recently developed numerical schemes are used in an integrated framework to simulate the plume flow from a representative solid propellant rocket at an altitude of $\mathbf{1 1 4}$ $\mathrm{km}$. In nonequilibrium regions the gas is modeled using the direct simulation Monte Carlo method, while a particle simulation method based on a simplified approximation of the Boltmann equation is used in near-equilibrium regions. Condensed phase $\mathrm{Al}_{2} \mathrm{O}_{3}$ particles, which are ejected with exhaust gases through the nozzle, are included in the simulation through a Lagrangian tracking scheme which allows for two-way interphase coupling of momentum and energy. Plume radiation properties are determined through a Monte Carlo ray trace model that incorporates effects of spectrally resolved emission, absorption and scattering. Radiation calculations are strongly coupled to the flowfield simulation, and allow particle emission and absorption to affect properties of both the particles and gas. Simulation results are compared with available experimental measurements, and generally good agreement is found.
\end{abstract}

\section{Introduction}

$T^{H}$

HE simulation of high altitude exhaust plumes from solid propellant rockets presents a number of modeling difficulties, and involves consideration of several complex physical processes which are not entirely understood. At very high altitudes (above roughly $100 \mathrm{~km}$ ) solid rocket motor (SRM) exhaust gases typically experience a high degree of thermal nonequilibrium through much of the plume. As the exhaust gases rapidly expand beyond the nozzle exit, thermal energy is increasingly distributed nonuniformly among the translational and internal degrees of freedom, and the gas velocity distribution may deviate significantly from the equilibrium limit. This is particularly true around the nozzle lip, in the plume backflow region upstream of the nozzle exit plane, and in the plumeatmosphere interaction region where a thick mixing layer may be bounded on either side by highly diffuse shock waves. ${ }^{1}$ Under these conditions the quasi-equilibrium assumptions underlying most continuum CFD methods break down, and alternate simulation techniques valid for transitional flow regimes must be used.

Additional procedures are required for inclusion of the $\mathrm{Al}_{2} \mathrm{O}_{3}$ particle phase, which consists of particles in both liquid and solid states with diameters ranging from 0.1 to $10 \mu \mathrm{m}$, and which commonly accounts for $15-30 \%$ of the mass flow through the nozzle. ${ }^{2}$ Momentum and energy exchange between particles and the surrounding gas may significantly influence properties of both phases, and the locally free molecular nature of gas-particle interactions makes a kinetic theory approach more appropriate than traditional continuum methods for two phase flow simulation. Coupling between radiative heat transfer and flowfield characteristics may also be important, as particle phase emission and absorption can significantly affect properties of both the particles and gas.

Over the course of a multi-year project, numerical modeling procedures have been developed for a number of potentially important phenomena associated with high altitude SRM plume flows, and various models have been individually tested on simple test cases and subscale flows involving a freely expanding plume. ${ }^{3-6}$ For the current effort, a number of these physical models are used together on a larger scale, more complicated flow which better represents the plume flows of interest. Detailed simulation results are presented, and a comparison is made between calculated radiation characteristics and available experimental data.

\footnotetext{
${ }^{1}$ Post-doctoral research fellow, AIAA member.

${ }^{2}$ Professor, AIAA associate fellow.
} 


\section{Simulation Setup and Modeling Procedures}

For ease of comparison and to limit the required computer resources, experimental measurements are desired for a plume flow which can be modeled through an axisymmetric simulation. The exhaust flow should therefore be expelled through a single nozzle, and the rocket should be oriented at a small inclination relative to its direction of motion. As found from an extensive literature search, the only experimental measurements for a single-nozzle high altitude SRM plume flow in the open literature are those of Erdman et al. ${ }^{7}$ In a flight experiment termed "Bow Shock Ultraviolet 2" (BSUV-2), onboard sensors were used to measure spectral radiance in the near-UV range (wavelengths of 0.2 to $0.4 \mu \mathrm{m}$ ) from upper stage SRM exhaust plumes at altitudes between roughly 100 and $120 \mathrm{~km}$. The plume from the third stage Star-27 SRM at an altitude of $114 \mathrm{~km}$ is used as a test case here, and while no experimental data is available for flowfield properties, we can to a limited extent infer overall simulation accuracy from a comparison of radiation characteristics between numerical and experimental results.

Following previous simulations of Candler et al. ${ }^{8}$ for the same flow at $114 \mathrm{~km}$, we perform a series of axisymmetric simulations on a rectangular grid which extends from $10 \mathrm{~m}$ upstream to $75 \mathrm{~m}$ downstream of the nozzle exit plane, and $30 \mathrm{~m}$ radially outward from the nozzle centerline. The nozzle exit diameter is $0.78 \mathrm{~m}$, and the rocket surface geometry is approximated as a blunted cone-cylinder shape of length $3.1 \mathrm{~m}$. The grid geometry is shown in Fig. (1).

\section{A. Gas phase calculations}

To account for the high degree of gas thermal nonequilibrium expected through much of the grid domain, the direct simulation Monte Carlo (DSMC) method is used to calculate gas properties in regions of relatively low density flow. ${ }^{9}$ The variable hard sphere (VHS) collision model is employed to approximate the variation in transport properties with temperature, and the Larsen-Borgnakke model ${ }^{9}$ is used together with a total collision energy model for the probability of inelastic collisions ${ }^{10}$ to determine energy exchange between translational and rotational modes.

In a typical SRM exhaust flow, the gas density in plume core regions downstream of the nozzle may be far too high to meet standard DSMC cell size requirements, so an alternate approach for gas phase simulation must be used in these regions. The traditional collision-limiter approach ${ }^{11}$ provides a simple solution, but is considerably less efficient and arguably less accurate than alternate methods which involve resampling some fraction of gas particle velocities during each time step from predetermined distribution functions. Both types of approaches can produce large artificial diffusion errors, and tend to enforce a near-equilibrium gas velocity distribution that may not be physically justified when cell dimensions are much greater than a mean free path. ${ }^{12}$ However, these approaches are generally far simpler to implement than alternate methods, such as hybrid CFD/DSMC algorithms, for simulating flowfields that include both continuum regions and nonequilibrium regions where a DSMC calculation is desired. This is particularly true for simulations involving a number of additional physical models, as is the case here, or those for which strong two-way coupling may exist between continuum and nonequilibrium regions.

For simulation of the BSUV-2 flow, gas properties in the high density, near-equilibrium plume core region are calculated using a recently developed particle method based on the ellipsoidal statistical Bhatnagar-Gross-Krook (ES-BGK) approximation of the Boltzmann equation. ${ }^{13}$ This method is intended for efficient simulation of moderately rarefied flows, and allows for variable-rate rotational relaxation effects in a gas mixture while enforcing exact conservation of mass, momentum and energy. The location of the boundary between DSMC and ES-BGK domains is determined using a standard cutoff value $(0.05)$ of a commonly used nondimensional continuum breakdown parameter. ${ }^{14}$ This parameter is calculated throughout the grid for an initial simulation where the ES-BGK method is applied to regions of both high and low gas density, and ES-BGK calculations are subsequently used only in regions where the condition for continuum breakdown is satisfied in the initial simulation. Domains for ES-BGK and DSMC methods are shown in Fig. (2). Note that nearly the entire plume region downstream of the nozzle exit is contained within the ES-BGK domain. We expect, however, that a larger portion of the plume would be within the DSMC domain if the grid were extended further downstream, where the reduced gas density should make nonequilibrium effects more prominent.

\section{B. Additional modeling procedures}

Condensed phase particles are included in the simulation here through a standard Lagrangian approach, where representative particles are tracked through the grid and sorted into cells using procedures similar to those in DSMC. The momentum and energy transfer to a particle from the surrounding gas is computed using a method of Gallis et

al., ${ }^{15}$ based on assumptions of locally free molecular flow and an approximation that all interphase collisions involve either specular reflection or diffuse reflection with full thermal accommodation to the particle temperature. The 
reciprocal momentum and energy transfer from a particle to the gas is determined through a probabilistic two-way coupling approach. ${ }^{3}$ Modifications to both methods are made to allow for nonspherical particles and to account for effects of particle rotation. ${ }^{4}$ A model for nonequilibrium crystallization of liquid $\mathrm{Al}_{2} \mathrm{O}_{3}$ droplets is implemented as well. ${ }^{5}$

Radiative heat transfer to and from the particles is calculated using a Monte Carlo ray trace (MCRT) radiation model, which involves tracking a large number of representative energy bundles through the computational domain. ${ }^{6}$ Energy bundles here represent a large collection of photons over some finite wavelength range (termed a "wave number bin" here for consistency with Ref. 6), and are created periodically at randomly selected source particles throughout the grid. Additional energy bundles are created along inflow boundaries at the nozzle exit, to account for effects of continuum searchlight emission from within the nozzle. The flux of energy bundles through userdesignated sensor surfaces is used to calculate spectral radiance at multiple simulated sensors positioned within or outside the grid. This model is strongly coupled to the flowfield simulation, and allows for spectrally resolved radiation calculations in an emitting, absorbing and scattering medium of arbitrary optical thickness. Particle emission and absorption characteristics may depend on particle size, temperature and wavelength, and anisotropic scattering is modeled using the Henyey Greenstein scattering phase function. ${ }^{16}$

\section{Boundary conditions}

Following simulations of Anfimov et al. ${ }^{17}$ for the same BSUV-2 flow, the $\mathrm{Al}_{2} \mathrm{O}_{3}$ particle phase is assumed to account for $30 \%$ of the mass flow rate at the nozzle exit, and is made up of seven discrete particle sizes ranging from 0.3 to $6 \mu \mathrm{m}$ in diameter. Properties for each particle size are independently specified along inflow boundaries at the nozzle exit plane. These inflow properties, as well as those of the gas, are taken from a nozzle flow simulation by the same authors.

A rough estimate of the initial solid mass fraction for each particle size is found by taking the difference between the nucleation temperature $(1930 \mathrm{~K})$ for homogeneous crystallization of $\mathrm{Al}_{2} \mathrm{O}_{3}$ and the assigned particle temperature, then multiplying this by the ratio of the specific heat to the latent heat of fusion for liquid $\mathrm{Al}_{2} \mathrm{O}_{3}$. Based on this procedure and the initial particle temperatures given by Anfimov et al., ${ }^{17}$ at the nozzle exit the largest particles are in a completely liquid state and the smallest particles have a liquid mass fraction of $58 \%$. Particle phase inflow properties are provided in Table (1).

The exhaust gas species are limited here to the primary species $\mathrm{H}_{2}, \mathrm{~N}_{2}$ and $\mathrm{CO}$, with initial mole fractions of 0.38, 0.31 and 0.31 respectively. At atmospheric inflow boundaries shown in Fig. (1), the gas has mole fractions of 0.78 for $\mathrm{N}_{2}$ and 0.22 for $\mathrm{O}_{2}$, with a temperature of $288 \mathrm{~K}$, a density of $5.79 \times 10^{-8} \mathrm{~kg} / \mathrm{m}^{3}$, and a freestream Mach number of 13.5. A thermal accommodation coefficient of 0.9 is used for collisions between gas molecules and condensed phase particles, while the outer surfaces of the rocket are modeled as diffusely reflecting walls at $300 \mathrm{~K}$ with full thermal accommodation.

\section{Plume radiation properties}

In the particle radiation model employed here, values of the absorption index $\mathrm{k}$ for condensed phase particles are used to calculate particle absorption and emission properties. The absorption index values are in turn calculated as a function of temperature and wavelength. Within the IR range where most radiative energy transfer through the plume is concentrated, absorption index values are taken from experimental measurements of Konopka, Reed and Calia. ${ }^{18}$ Based on this data, radiation calculations here discretize the measured IR region into 10 wave number bins, corresponding to wavelengths between 1.0 and $4.5 \mu \mathrm{m}$. Of the two SRM exhaust flows from which particles were collected and investigated by these authors, the second (rocket 2) was found to give k values more in line with other experimental data and correlations in the literature. ${ }^{19}$ Values calculated from SEM measurements for the second flow are therefore used here.

To demonstrate the degree of variation in $\mathrm{k}$ with both wavelength and temperature, measured $\mathrm{k}$ values of Konopka, Reed and Calia ${ }^{18}$ are plotted in Fig. (3) as a function of wavelength for two different particle temperatures. Lines in the figure labeled as "standard" denote data for the second rocket, while lines labeled "alternate" give k values measured for the first. Note that, through much of the wavelength range considered, the two sets of data points differ by roughly an order of magnitude. As discussed by these authors and by Reed and Calia, ${ }^{2}$ this difference may be attributed primarily to the large influence of lattice defects and impurities on the radiative properties of solid $\mathrm{Al}_{2} \mathrm{O}_{3}$, which tend to produce a significant and unavoidable uncertainty in results from SRM plume radiation calculations. 
For comparison with the experimental plume radiance measurements of Erdman et al., ${ }^{7}$ radiation calculations must include UV-range wavelengths between 0.2 and $0.4 \mu \mathrm{m}$. While very few data sets exist for $\mathrm{Al}_{2} \mathrm{O}_{3}$ absorption index values in this range, the correlations employed in plume calculations of Anfimov et al. ${ }^{17}$ are stated as valid over the UV wavelengths of interest. These correlations involve a summation over four different intrinsic emission/absorption mechanisms, and as in the data of Konopka, Reed and Calia, ${ }^{18} \mathrm{k}$ values here depend on both wavelength and temperature but are independent of the particle phase composition. Plots of the UV absorption index as a function of wavelength are shown in Fig. (4) for three different particle temperatures, based on these correlations. (The two additional lines in the figure - those for constant emissivity and constant absorption index are discussed below in Section III.)

To incorporate UV radiation calculations into the plume simulation, an additional eight wave number bins are added to cover the wavelength range between 0.2 and $1.0 \mu \mathrm{m}$, with most of these bins concentrated toward smaller wavelengths $(0.2$ to $0.4 \mu \mathrm{m})$ for which experimental plume radiance values are available. Absorption index values over this entire range are taken from the correlations of Anfimov et al. ${ }^{17}$

While particle absorption and emission properties are assumed here to vary with the particle absoption index, or the imaginary part of the index of refraction, the radiation model uses the real part $n$ of the index of refraction to determine scattering characteristics. For all 18 wave number bins over the wavelength range between 0.2 and 4.5 $\mu \mathrm{m}$, values of $\mathrm{n}$ are computed as a function of the wave number $\eta_{\mathrm{i}}$ at the center of each bin through a correlation given by Duval, Soufiani and Taine ${ }^{19}$

$$
\mathrm{n}=\left(0.9904+2.02 \times 10^{-5} \mathrm{~T}_{\mathrm{p}}\right)\left[1+\frac{1.024}{1-0.00376 \eta_{\mathrm{i}}^{2}}+\frac{1.058}{1-0.01225 \eta_{\mathrm{i}}^{2}}+\frac{5.281}{1-321.4 \eta_{\mathrm{i}}^{2}}\right]^{1 / 2}
$$

where the weak temperature dependence in Eq. (1) is neglected by assuming a particle temperature $T_{p}$ of $2000 \mathrm{~K}$. Following Reed et al., ${ }^{20}$ the average cosine of the scattering angle in the Henyey Greenstein scattering phase function ${ }^{16}$ is set to 0.5 , and an effective temperature of $1300 \mathrm{~K}$ is used for searchlight emission at the nozzle exit.

\section{E. Additional simulation parameters}

Spectral radiance calculations are made for two different simulated sensors. The first of these is positioned near the rocket surface, with its center $2 \mathrm{~m}$ upstream of the nozzle exit plane and $0.6 \mathrm{~m}$ from the central axis. The conical viewing region has a maximum zenithal angle of $2^{\circ}$, and is centered along a line oriented at a $4^{\circ}$ inclination to the axis. The sensor position and direction are set here for comparison between calculated radiance values and measurements taken through a periscope-mounted onboard radiometer in the BSUV-2 flight experiment. ${ }^{7}$

An additional simulated sensor is positioned outside the grid domain, and is used to characterize plume emission as would be measured from ground or air-based remote sensors. (No such measurements were made for the BSUV-2 flight.) This second sensor is centered at a point $50 \mathrm{~m}$ downstream of the nozzle exit and $100 \mathrm{~m}$ radially outward from the axis, with a conical viewing region oriented normal to the axis and bounded by the zenithal angle $\omega=10^{\circ}$. Locations and viewing regions for both sensors are shown in Fig. (5).

All models described above have been implemented and individually tested on a modified version of the DSMC code MONACO. ${ }^{21}$ Calculations are run for approximately 60 hours on eight $1.4 \mathrm{GHz}$ AMD Athlon processors. Domain decomposition is used to roughly evenly divide the computational load among all processors, and cell size, time step and numerical weights are carefully varied throughout the grid to balance overall simulation accuracy with computational efficiency. The unstructured grid is made up of about 87,000 triangular cells, through which around 7.5 million DSMC gas particles and 160,000 representative $\mathrm{Al}_{2} \mathrm{O}_{3}$ particles are tracked at steady state.

\section{Simulation Results}

Some general characteristics of the simulated flowfield are displayed in Fig. (6), which gives a close-up view of gas bulk velocity contours and streamlines in a $10 \mathrm{~m}$ long region surrounding the rocket. The figure shows a highly diffuse bow shock, with a large boundary layer along the front portion of the rocket surface. Further downstream, a thick mixing layer forms along the region of plume-atmosphere interaction, and a small plume backflow region is found just upstream of the nozzle exit and within a few meters of the rocket. Within the plume, streamlines and velocity contours show the characteristics of a near-free expansion flow - including a complete absence of shocks - 
as expected for a highly underexpanded plume at very high altitude. ${ }^{1}$ Note the well resolved boundary between the plume and atmospheric flow regions.

\section{A. Gas and particle mass density}

Contours of mass density throughout the grid are shown in Fig. (7). Particle phase mass density is displayed in the upper half of the figure, while the lower half gives contours of gas density. Note that particles are only found in roughly half of the simulation domain, as the maximum divergence angle for the particle phase is restricted by particle mass. Within much of the region where particles are found, the particle mass density is shown to continuously increase with distance from the axis. This trend can be explained as follows: In the plume nearfield region just beyond the nozzle exit, particles are forced outward from the centerline by the expanding gas. As the drag force on a particle ${ }^{15}$ scales with the square of the particle diameter $D_{p}$ and the particle mass is proportional to $\mathrm{D}_{\mathrm{p}}{ }^{3}$, the radial acceleration of an individual particle in this region will vary as roughly $1 / \mathrm{D}_{\mathrm{p}}$. Thus, in a given radial plane, smaller particles will be found over a range which extends further from the axis. As shown in the top half of Fig. (7), this results in a gradual decrease in particle mass density with distance from the axis, due to the presence of a range of particle sizes.

The variation in maximum divergence angle with particle size is further shown in Fig. (8), which displays the spatial variation in characteristic particle dimensions throughout the grid. The upper half of the figure shows contours of the average particle diameter, while the lower half gives contours of the Sauter mean diameter. (The Sauter mean diameter is a commonly used particle size parameter, and is defined as the diameter of a particle with a volume-to-surface area ratio equal to the ratio of total volume to total surface area among all particles within a differential control volume.) Although both the upper and lower portions of Fig. (8) show a continual decrease in the characteristic particle size with distance from the axis through much of the grid, the slope of this decrease is not constant due to the use of a discrete particle size distribution.

As the dimensions of the simulation domain are about two orders of magnitude greater than the nozzle exit radius, particle characteristics observed in both Figs. (7) and (8) primarily reflect trends in the farfield region where interphase momentum transfer can be assumed negligible. The particles move along nearly straight trajectories far from the nozzle, so the contour lines shown projecting from the nozzle exit can be assumed straight if we neglect effects of statistical scatter and interpolation between cell centers.

The gas density is shown in the lower half of Fig. (7) to decrease continuously with downstream distance through much of the simulation domain, as is expected for any highly underexpanded plume flow. A sharp upward spike in gas density is observed in the plume-atmosphere interaction region, where a diffuse shock forms as a result of both the presence of a solid body and the convergence of streamlines between areas occupied by atmospheric and exhaust gas species. The reduction in gas density found near the central axis is a non-physical characteristic of axisymmetric simulations using particle methods; to populate cells near the axis with a sufficient number of gas particles, the numerical weight of particles in these regions will often need to be reduced. This in turn leads to random walk errors, which tend to decrease the accuracy of simulation results at points near the axis.

\section{B. Particle temperatures}

Contours of average particle temperature are shown in Fig. (9) for two different sizes of $\mathrm{Al}_{2} \mathrm{O}_{3}$ particles. The upper half of the figure gives temperatures for $0.4 \mu \mathrm{m}$ diameter particles, which are near the lower end of the particle size distribution, while temperature contours in the lower half of the figure are for much larger particles of 4 $\mu \mathrm{m}$ diameter. Note the difference in the regions occupied by particles of the two different sizes. As discussed above, smaller particles experience a greater radial acceleration in the plume nearfield region just beyond the nozzle exit, so the maximum divergence angle is greater for the smaller particle size. Also note that the temperature of the smaller $0.4 \mu \mathrm{m}$ particles is uniformly shown to be about $1200 \mathrm{~K}$ lower than that of the $4 \mu \mathrm{m}$ particles. This follows primarily from the relation between particle heat capacity, which scales as $\mathrm{D}_{\mathrm{p}}{ }^{3}$, and the convective heat transfer rate, which is proportional to $\mathrm{D}_{\mathrm{p}}{ }^{2}$. It can be shown from these scaling relations that the rate of change in particle temperature tends to vary as $1 / D_{p}$, so that smaller particles will be more rapidly cooled by the surrounding gas in the plume nearfield region where convective heat transfer is significant.

For both particle sizes in Fig. (9), the average temperature is generally found to increase with distance from the central axis. This is due to the fact that, on the scale of the simulation domain, most trends in particle temperature are dominated by the influence of convective heat transfer. As the convective heat transfer rate is proportional to the local gas density, we expect that particles which pass through the lower-density portions of the plume nearfield region further from the axis will experience less heat loss to the cooler surrounding gas. Particles further from the 
axis will therefore retain higher temperatures far downstream, as is shown in the figure. The increase in particle temperatures at points very close to the axis is also related to the radial variation in gas density within the plume nearfield region. As explained in the above discussion of Fig. (7), random walk errors in cells near the axis produce an artificially low gas density, which in turn reduces convective heat transfer rates for particles which pass through these cells.

Most of the streamwise reduction in temperature shown for both particle sizes in Fig. (9) is a result of radiative heat loss. Due to the large scale of the simulation domain in relation to the size of the rocket, the regions where convective heat transfer and phase change make significant contributions to the particle energy balance are restricted to a relatively small area, within a few meters of the nozzle exit. A close-up view of average particle temperatures in this area, again for particles of diameter 0.4 and $4 \mu \mathrm{m}$, is shown in Fig. (10).

Note the contrast between Figs. (9) and (10) in the magnitude of observed temperature variation with downstream distance for both particle sizes. Particle temperatures are found to vary far more rapidly in the plume nearfield region displayed in Fig. (10) than in regions further downstream, shown in Fig. (9), which make up most of the simulation domain. This difference in temperature gradients is due primarily to the rapid reduction in gas density with downstream distance. As shown in Fig. (7), the gas density is far higher in the nearfield plume region just downstream of the nozzle than elsewhere in the plume, so convective heat transfer rates will be far higher as well near the nozzle exit.

\section{Particle phase change}

A close observation of $4 \mu \mathrm{m}$ particle temperature contours in Fig. (10) reveals a rapid temperature jump about $0.4 \mathrm{~m}$ downstream of the nozzle exit. This jump is associated with the initiation of the phase change process, and results from the release of the latent heat of fusion for liquid $\mathrm{Al}_{2} \mathrm{O}_{3}$. As given in Table (1), $4 \mu \mathrm{m}$ particles are assumed to be in a completely liquid state at inflow boundaries along the nozzle exit, and are rapidly cooled by the surrounding gas until they reach the nucleation temperature $(1930 \mathrm{~K})$ for homogeneous crystallization. ${ }^{5}$ Once this temperature is reached, a radially symmetric crystallization front progresses from the surface of a particle toward its center. The heat release associated with the phase change process causes a sudden increase in particle temperature, and this temperature increase in turn slows the progress of the crystallization front. For some time a quasiequilibrium state may be reached, where the heat release during crystallization is balanced by convective and radiative heat loss, and the particle maintains a nearly uniform temperature. Further downstream, as the liquid mass fraction approaches zero and the rate of heat release is reduced, the particle temperature again begins to decrease as observed in Fig. (10).

To demonstrate the correlation between phase change and the variation in particle temperatures, a contour plot of the liquid mass fraction is included as Fig. (11). The upper half of the figure shows cell-averaged liquid mass fractions for $4 \mu \mathrm{m}$ particles, while the lower half gives contours for even larger particles of $6 \mu \mathrm{m}$ diameter. (As given in Table (1), most particles of smaller sizes are assumed to be in a completely solid state at the nozzle exit, so crystallization processes occur within the plume only for the larger particles.) A comparison of characteristics for 4 $\mu \mathrm{m}$ particles between Figs. (11) and (10) shows that, as described above, the particle temperature jump about $0.4 \mathrm{~m}$ downstream of the nozzle exit is accompanied by the initiation of phase change and a rapid reduction in the liquid mass fraction. Particle temperatures level off further downstream, as the gradient in the liquid mass fraction decreases, and the particles ultimately approach a completely solid state.

In comparing liquid mass fraction contours for the two different particle sizes shown in Fig. (11), we find that phase change begins significantly further downstream for the larger $6 \mu \mathrm{m}$ particles. As explained above, the rate of temperature decrease due to convective heat transfer tends to vary as roughly the inverse of the particle diameter, so that larger particles will experience a longer residence time in a completely liquid state before reaching the nucleation temperature at which crystallization may begin.

For both 4 and $6 \mu \mathrm{m}$ particles, the onset of crystallization is shown in Fig. (11) to occur progressively downstream for particles which move along trajectories further from the axis. As with the radial variation in particle temperatures, this trend is a result of the decrease in gas density with distance from the axis. Particles which pass through regions of lower gas density will cool more slowly through convective heat transfer, so these particles tend to reach the nucleation temperature at points further downstream.

\section{Convective and radiative heat transfer rates}

Figure (12) shows the variation with downstream distance in the magnitude of mean convective and radiative heat transfer rates, as calculated per particle and averaged over all particle sizes. Values are extracted along a line 
which runs very close to the central axis, but is sufficiently far from the axis to be relatively unaffected by random walk errors associated with the variation in numerical weights. Both heat transfer rates in the figure are negative through the entire simulation domain, as particles throughout the plume are, on average, losing energy to their surroundings through both convective and radiative heat transfer.

The radiative heat transfer rate is shown to be relatively constant with downstream distance, due to an overall gradual variation in particle temperatures and the fact that radiative absorption is found to have a comparatively small effect. Much of the variation in radiative transfer observed on the plot is a consequence of statistical scatter, as results from the small number of representative particles which pass through cells bordering the axis. However, a significant increase in the radiative transfer rate is found about $0.4 \mathrm{~m}$ downstream of the nozzle exit. This may be attributed to the temperature jump associated with the onset of phase change in $4 \mu \mathrm{m}$ particles, which account for roughly $60 \%$ of the total $\mathrm{Al}_{2} \mathrm{O}_{3}$ mass within the plume.

The slow reduction in radiative heat transfer observed to begin about $10 \mathrm{~m}$ downstream of the nozzle is also a result of the variation in particle temperature. As particle temperatures decrease due to radiative heat loss in the farfield plume region, the magnitude of the radiative energy transfer rate will decrease as well, due to the strong temperature dependence in particle emissive power.

In contrast to the relatively uniform rate of radiative heat transfer, the mean convective heat transfer rate is shown in Fig. (12) to decrease rapidly with downstream distance. The spatial variation in convective heat transfer occurs mainly as a result of the large downstream decrease in gas density. As the gas approaches a free molecular state far from the nozzle, the gas density will decrease along the centerline as the inverse square of the distance from the nozzle exit. The farfield convective heat transfer rate will therefore have approximately the same inverse square variation, as is shown in the figure. Note that, at points along the centerline beyond about $6 \mathrm{~m}$ (or 8 nozzle exit diameters) downstream of the nozzle exit, radiative emission becomes the dominant mechanism for energy transfer between a particle and its surroundings.

\section{E. Radiative energy flux}

In Fig. (13), contours are shown for the direction-averaged spectral radiative energy flux $E_{\lambda}$ at wavelengths of $2.2 \mu \mathrm{m}$ and $0.24 \mu \mathrm{m}$. This flux is defined as the rate per unit wavelength at which photons within a narrow wavelength range impinge on a spherical surface, divided by the cross sectional area of the sphere. While $E_{\lambda}$ values have little physical significance outside of the radiation model employed here, ${ }^{6}$ contours of $E_{\lambda}$ provide a convenient means of quantifying the variation in spectrally resolved radiation intensity throughout the simulated flowfield. The upper half of Fig. (13) can therefore be viewed as a measure of IR radiation intensity through the grid, while the lower half shows the intensity at a representative wavelength in the UV range. In comparing $E_{\lambda}$ contours for the two different wavelengths, we find that the IR radiation intensity is consistently about four orders of magnitude greater than the UV intensity, as follows from the fact that thermal radiative emission at the particle temperatures shown in Figs. (9) and (10) is typically concentrated in the IR range. ${ }^{2}$

A significant difference between the two wavelengths is also found in the shape of contour lines shown in Fig. (13). This difference may be attributed to the variation with wavelength in the temperature dependence for thermal emission. At smaller wavelengths, the spectral emissive power for a blackbody tends to depend far more strongly on the temperature of the body. ${ }^{22}$ This characteristic is preserved in the particle radiation model, through formulas used to calculate the emissive power output of a representative source particle. ${ }^{6}$ As shown in Fig. (9), particles further from the axis tend to have higher temperatures, so experience a greater rate of radiative heat loss. This temperature difference has a stronger correlation to emissive power in the UV range than in the IR range, so a more pronounced ridge is shown in the UV energy flux contours near the location of higher temperature particles.

As shown in Fig. (13), the energy flux for both wavelengths is greatest at the nozzle exit, due primarily to the corresponding maximum in particle mass density and a reduction in the intensity of searchlight emission with distance from the nozzle. Particles are modeled here as volumetric emitters, ${ }^{2}$ so the magnitude of the radiative energy flux should scale roughly with the local particle mass density. As this density decreases with downstream distance due to the divergence of particle trajectories through the plume, a continuous reduction in radiative energy flux is found in the axial direction.

A reduction in energy flux is also observed in the radial direction, particularly outside the region where particles are found. This follows from the fact that, in an axisymmetric simulation, the ratio of cell volume to the projected area on the grid will scale with distance from the axis. The average number of energy bundles which pass through a cell is proportional to the projected area of that cell, and the contribution of each bundle to the energy flux is proportional to the inverse of the cell volume. We therefore expect the net energy flux through cells outside the 
particle domain to scale approximately with the inverse of the distance from the axis, as is shown in both the upper and lower portions of Fig. (13).

\section{F. Plume spectral radiance}

In Fig. (14), values are presented for the plume spectral radiance $I_{i}$ as observed at the onboard and remote simulated sensors shown in Fig. (5). Values in Fig. (14) are averaged over results from eight independent simulations. In each of these simulations the random number generator is initialized at a different point, but all are otherwise identical. The error bars for the onboard sensor values give 95\% confidence intervals based on a wavelength-averaged standard deviation $\sigma_{\text {avg, }}$ from the eight simulations, and show that the stochastic nature of the radiation model produces significant scatter in these values. Error bars for radiance values from the remote sensor are too small to include in the figure. (The variation in scatter between the two sets of values is mainly the result of differences in sensor surface area, viewing angle and angular resolution as shown in Fig. (5).)

To determine the height of the error bars shown in Fig. (14), we assume a Gaussian distribution of radiance values among the eight simulations for each wave number bin $i$, and we further assume that the ratio of the standard deviation $\sigma_{\mathrm{i}}$ to the mean radiance $\mathrm{I}_{\mathrm{i}}$ from the eight simulations is independent of wavelength. It follows from these assumptions that error bars for a $95 \%$ confidence interval should be spaced a distance of about $1.96 \sigma_{\text {avg, }}$ from the corresponding mean value $\mathrm{I}_{\mathrm{i}}$, where $\sigma_{\text {avg, } \mathrm{i}}=\mathrm{I}_{\mathrm{i}}\left\langle\sigma_{\mathrm{j}} / \mathrm{I}_{\mathrm{j}}\right\rangle$ and the operator \langle\rangle denotes an arithmetic average over all wave number bins. ${ }^{23}$

Both data sets plotted in Fig. (14) display a relatively small wavelength dependence through much of the IR range, and a sharp drop-off in the visible and UV regions. These trends, as well as the general shape of the radiance profiles, are consistent with Planck's solution for the spectral distribution of blackbody emissive power. It can further be shown through Wien's displacement law that the wavelength of maximum radiance for both sensors, around $0.8 \mu \mathrm{m}$, corresponds to a blackbody temperature of roughly $3600 \mathrm{~K}^{22}$ While no particles in the simulation actually reach that temperature, we expect that, if a greater spectral resolution is used in radiation calculations and if the wavelength dependence in particle emissivity is disabled, the corresponding blackbody temperature will be significantly reduced.

As mentioned above in the discussion of Fig. (5), the location and orientation of the simulated onboard sensor corresponds to that of a periscope-mounted radiometer used to measure UV spectral radiance during the BSUV-2 flight experiment. ${ }^{7}$ These measured values are presented in Fig. (15) along with corresponding simulation results in the wavelength range between 0.2 and $0.4 \mu \mathrm{m}$. Published numerical results of Candler et al. ${ }^{8}$ and Anfimov et al. ${ }^{17}$ are also included for comparison. Both of these previous simulations employ continuum CFD methods which for this flow may be less appropriate than the kinetic approach used here, particularly in the highly rarefied regions within the DSMC domain shown in Fig. (2). Still, somewhat better agreement is found with experiment for these results than for those from the present simulations, particularly at wavelengths below around $0.25 \mu \mathrm{m}$. Note however that in the simulations of Candler et al., ${ }^{8}$ particle phase enthalpies at the nozzle exit were selected for best agreement between calculated and experimental radiance values at the $0.23 \mu \mathrm{m}$ wavelength. This procedure effectively rescales the UV radiance profile to match experimental results, and reduces the utility of any quantitative comparison with experiment.

In comparing the level of agreement between the different sets of results, also note that radiance values determined by Anfimov et al. ${ }^{17}$ are subject to uncertainty associated with correlations for the particle absorption index introduced by these same authors. The correlations exhibit a strong temperature and wavelength dependence that has not been verified elsewhere in the literature, and they do not account for extrinsic properties (such as impurity concentrations and lattice defects) that have been found to dominate radiation characteristics of solid phase particles. $^{2}$ As the same correlations are employed for the simulations presented here, the resulting uncertainty in radiance values for both the new simulations and those of Anfimov et al. leads to some doubt regarding the validity of using these values to assess overall simulation accuracy.

To determine the effect of the UV absorption index correlations on radiance values, two additional simulations are performed. In the first simulation, the absorption index is given a constant value of 0.01 through the UV range. As shown in Fig. (4), this value roughly characterizes an average of values taken from the correlations of Anfimov et al. ${ }^{17}$ over a range of temperatures and wavelengths. In the second simulation the UV absorption index is set so that, for a given particle size, the spectral emissivity $\varepsilon_{\lambda}$ in the UV range is constant. In procedures employed here for plume radiation calculations, $\mathrm{Al}_{2} \mathrm{O}_{3}$ particle emissivities are determined as a function of the absorption index $\mathrm{k}$, the particle radius $R_{p}$ and the wavelength $\lambda$ 


$$
\varepsilon_{\lambda}=\frac{4 \mathrm{kR}}{\lambda}
$$

using a correlation of Reed and Calia ${ }^{2}$ based on Mie theory calculations of Plass. ${ }^{24}$ It follows from Eq. (2) that, for a constant UV spectral emissivity and a given particle radius, the absorption index in the UV range must be proportional to the wavelength.

Particles are modeled through Eq. (2) as volumetric emitters, ${ }^{2}$ so that in an isothermal enclosure containing particles with a range of sizes, the emissive heat flux per unit volume will scale with the mean-cubed particle radius. As this flux should also scale with the average over all particle sizes of the quantity $\varepsilon_{\lambda} \mathrm{R}_{\mathrm{p}}{ }^{2}$, an effective emissivity which is independent of the particle radius must be proportional to the ratio of the mean-cubed radius to the meansquared radius. This ratio is equal to one-half the Sauter mean diameter, which may be substituted for $R_{p}$ in Eq. (2) for use with an effective emissivity which is independent of particle size.

Thus, to allow for values of the UV absorption index which give a uniform effective emissivity, we assume a characteristic Sauter mean diameter of $3.2 \mu \mathrm{m}$ (as is found through much of the plume region occupied by particles) and use an emissivity of 0.2. From Eq. (2), this corresponds to the relation $k=0.031 \lambda$. As shown in Fig. (4), the resulting $\mathrm{k}$ values for this "constant emissivity" case are generally of the same order as those found through the correlations of Anfimov et al., ${ }^{17}$ and are very much in line with values used in the simulation involving a constant absorption index.

Radiance values from both additional simulations are shown in Fig. (15), and exhibit significantly better agreement with experiment than do results from simulations employing the UV absorption index correlations of Anfimov et al. ${ }^{17}$ In particular, the constant emissivity simulation gives excellent agreement with the experimental data at smaller wavelengths where the correlations of Anfimov et al. produce the greatest disagreement. While the choice of constant emissivity or absorption index values is to some extent arbitrary, the resulting consistency with experiment gives us reasonable confidence in the overall simulation accuracy.

\section{Summary and Conclusions}

A number of recently developed modeling procedures have been applied to the simulation of a representative SRM plume flow at high altitude. Simulations are performed to evaluate flowfield and radiation characteristics for the BSUV-2 flight experiment at an altitude of $114 \mathrm{~km}$. The gas is modeled using the DSMC method in atmospheric and plume-atmosphere interaction regions, while higher density regions within the plume are handled by an alternate particle method for gas flow simulation. This second method is based on the ES-BGK approximation of the Boltzmann equation, and accurately models moderate translational nonequilibrium while allowing for effects of a finite rotational relaxation rate. Condensed phase $\mathrm{Al}_{2} \mathrm{O}_{3}$ particles are included in the simulation through a Lagrangian modeling procedure. This procedure includes two-way coupled momentum and energy transfer between particles and the surrounding gas, and incorporates effects of nonequilibrium droplet crystallization. The included particle radiation model is strongly coupled to the flowfield simulation, and allows for spectrally resolved absorption, emission and anisotropic scattering in a medium of arbitrary optical thickness.

Detailed results are presented from the BSUV-2 flow simulation, and a comparison is made between calculated radiance values and experimental measurements. Relatively good agreement is observed here between simulation and experiment. This comparison can, to a limited extent, be used to evaluate the overall accuracy of the simulation procedures. However, the inherent uncertainty in inflow conditions and input parameters for the radiation model, as well as a complete lack of experimental data on flowfield properties, lead to some doubt in the adequacy of the evaluation method. As no other experimental results are available for a similar plume flow, the comparison of UV spectral radiance profiles presented here seems to be a reasonable, if not completely sufficient, method by which to assess the overall accuracy of the simulation scheme. While the inherent complexity and challenges assiociated with simulating this type of flow leave extensive room for future progress, it is hoped that the models and techniques employed here represent significant progress in the development of capabilities for high altitude SRM plume simulation.

\section{References}

\footnotetext{
${ }^{1}$ Simmons, F. S., Rocket Exhaust Plume Phenomenology, Aerospace Press, El Segundo, CA, 2000.

${ }^{2}$ Reed, R. A., and Calia, V. S., "Review of Aluminum Oxide Rocket Exhaust Particles,” AIAA Paper 93-2819,
} 1993. 
${ }^{3}$ Burt, J. M., and Boyd, I. D., "Development of a Two-Way Coupled Model for Two Phase Rarefied Flows," AIAA Paper 2004-1351, 2004.

${ }^{4}$ Burt, J. M., and Boyd, I. D., "Particle Rotation Effects in Rarefied Two Phase Plume Flows," 24th International Symposium on Rarefied Gas Dynamics, Monopoli, Italy, 2004.

${ }^{5}$ Burt, J. M., and Boyd, I. D., "Monte Carlo Simulation of a Rarefied Multiphase Plume Flow," AIAA Paper 2005-0964, 2005.

${ }^{6}$ Burt, J. M., and Boyd, I. D., “A Monte Carlo Radiation Model for Simulating Rarefied Multiphase Plume Flows,” AIAA Paper 2005-4691, 2005.

${ }^{7}$ Erdman, P. W., Zipf, E., Espy, P., Howlett, C., Levin, D. A., and Candler, G. V., "In-Situ Measurements of UV and VUV Radiation from a Rocket Plume and Re-entry Bow Shock,” AIAA Paper 92-0124, 1992.

${ }^{8}$ Candler, G. V., Levin, D. A., Brandenburg, J., Collins, R., Erdman, P., Zipf, E., Howlett, C., "Comparison of Theory with Plume Radiance Measurements from the Bow Shock Ultraviolet 2 Rocket Flight,” AIAA Paper 920125, 1992.

${ }^{9}$ Bird, G. A., Molecular Gas Dynamics and the Direct Simulation of Gas Flows, Clarendon Press, Oxford, 1994.

${ }^{10}$ Boyd, I. D., "Analysis of Rotational Noequilibrium in Standing Shock Waves of Nitrogen," AIAA Journal, Vol. 28, No. 11, 1990, pp. 1997-1999.

${ }^{11}$ Bartel, T. J., Sterk, T. M., Payne, J. L., and Preppernau, P., "DSMC Simulation of Nozzle Expansion Flow Fields,” AIAA Paper 94-2047, 1994.

${ }^{12}$ Breuer, K. S., Piekos, E. S., and Gonzales, D. A., “DSMC Simulations of Continuum Flows,” AIAA Paper 952088, 1995.

${ }^{13}$ Burt, J. M., and Boyd, I. D., "Evaluation of a Particle Method for the Ellipsoidal Statistical Bhatnagar-GrossKrook Equation,” AIAA Paper 2006-0989, 2006.

${ }^{14}$ Boyd, I. D., Chen, G., and Candler, G. V., "Predicting Failure of the Continuum Fluid Equations in Transitional Hypersonic Flows,” Physics of Fluids, Vol. 7, No. 1, 1995, pp. 210-219.

${ }^{15}$ Gallis, M. A., Torczynski, J. R., and Rader, D. J., "An approach for Simulating the Transport of Spherical Particles in a Rarefied Gas Flow via the Direct Simulation Monte Carlo Method," Physics of Fluids, Vol. 13, No. 11, 2001, pp. 3482-3492.

${ }^{16}$ Everson, J., and Nelson, H. F., "Development and Application of a Reverse Monte Carlo Radiative Transfer Code for Rocket Plume Base Heating,” AIAA Paper 93-0138, 1993.

${ }^{17}$ Anfimov, N. A., Karabadjak, G. F., Khmelinin, B. A., Plastinin, Y. A., and Rodinov, A. V., "Analysis of Mechanisms and Nature of Radiation from Aluminum Oxide in Different Phase States in Solid Rocket Exhaust Plumes," AIAA Paper 93-2818, 1993.

${ }^{18}$ Konopka, W. L., Reed, R. A., and Calia, V. S., "Measurements of Infrared Optical Properties of $\mathrm{Al}_{2} \mathrm{O}_{3}$ Rocket Particles,” AIAA Paper 83-1568, 1983.

${ }^{19}$ Duval, R., Soufiani, A., and Taine, J., "Coupled Radiation and Turbulent Multiphase Flow in an Aluminised Solid Propellant Rocket Engine," Journal of Quantitative Spectroscopy and Radiative Transfer, Vol. 84, No. 4, 2004, pp. 513-526.

${ }^{20}$ Reed, R. A., Beale, K. S., Neese, D. W., Sherrell, F. G., Roberds, D. W., and Oliver, S. M., "The Effect of Seachlight Emission on Radiation from Solid Rocket Plumes,” AIAA Paper 92-2918, 1992.

${ }^{21}$ Dietrich, S., and Boyd, I. D., "Scalar and Parallel Optimized Implementation of the Direct Simulation Monte Carlo Method,” Journal of Computational Physics, Vol. 126, 1996, pp. 328-342.

${ }^{22}$ Siegel, R., and Howell, J. R., Thermal Radiation Heat Transfer, Hemisphere Publishing, Washington, 1981.

${ }^{23}$ Hogg, R. V., and Tanis, E. A., Probability and Statistical Inference, Prentice Hall, 1997.

${ }^{24}$ Plass, G. N., "Temperature Dependence of the Mie Scattering and Absorption Cross Sections for Aluminum Oxide,” Applied Optics, Vol. 4, No. 12, 1965, pp. 1616-1619. 
Table 1. Particle properties at the nozzle exit.

\begin{tabular}{|l|l|l|l|l|}
\hline $\begin{array}{l}\text { Diameter, } \\
\mu \mathrm{m}\end{array}$ & $\begin{array}{l}\text { Mass flux, } \\
\mathrm{kg} / \mathrm{m}^{2} \mathrm{~s}\end{array}$ & $\begin{array}{l}\text { Temperature, } \\
\mathrm{K}\end{array}$ & $\begin{array}{l}\text { Speed, } \\
\mathrm{m} / \mathrm{s}\end{array}$ & $\begin{array}{l}\text { Liquid mass } \\
\text { fraction }\end{array}$ \\
\hline 0.3 & 0.0443 & 1562 & 2992 & 0.579 \\
\hline 0.4 & 0.0367 & 1634 & 3051 & 0.661 \\
\hline 0.6 & 0.133 & 1834 & 3023 & 0.89 \\
\hline 1 & 0.592 & 2293 & 2973 & 1 \\
\hline 2 & 2.29 & 1920 & 2855 & 0.989 \\
\hline 4 & 7.53 & 2178 & 2674 & 1 \\
\hline 6 & 1.84 & 2407 & 2472 & 1 \\
\hline
\end{tabular}

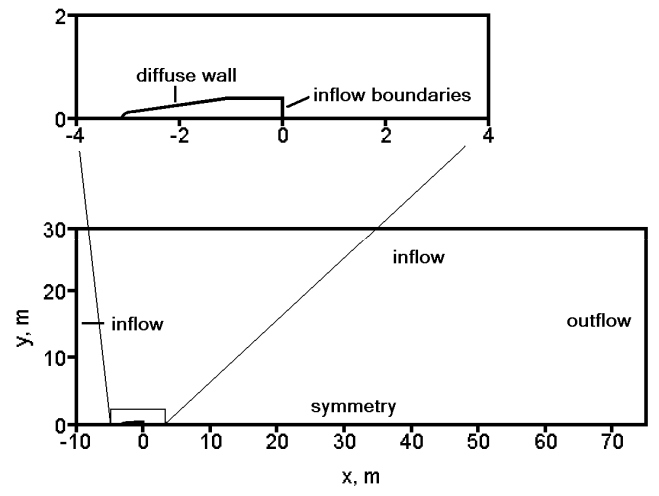

Fig. 1 Grid geometry for simulations of the BSUV-2 flow.

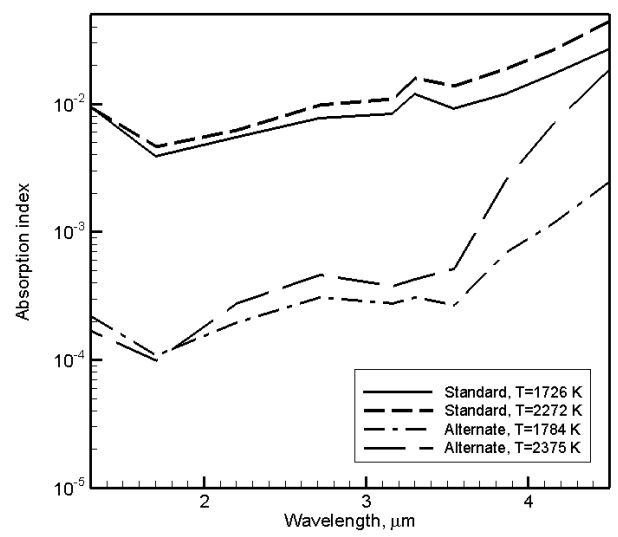

Fig. 3 Measured IR absorption index values for $\mathrm{Al}_{2} \mathrm{O}_{3}$.

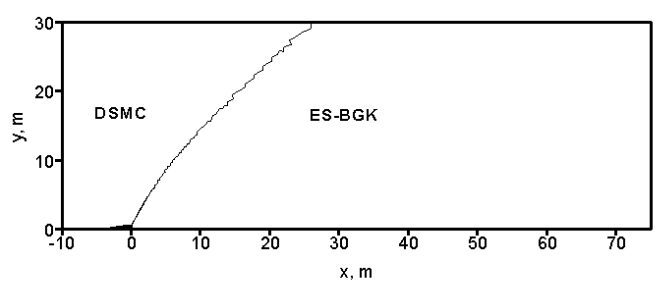

Fig. 2 Domains for DSMC and ES-BGK simulation methods.

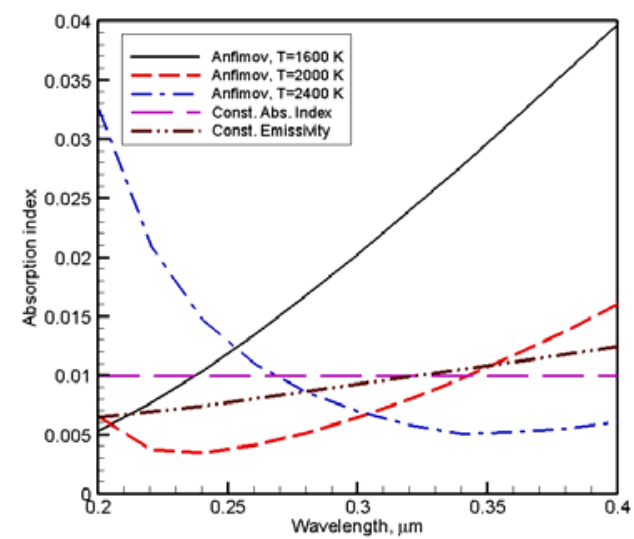

Fig. $4 \mathrm{UV}$ absorption index values for $\mathrm{Al}_{2} \mathrm{O}_{3}$. 


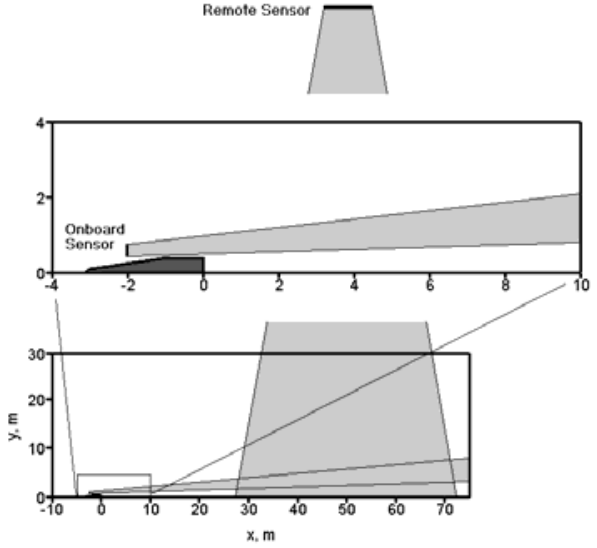

Fig. 5 Sensor viewing regions for plume radiance calculations.

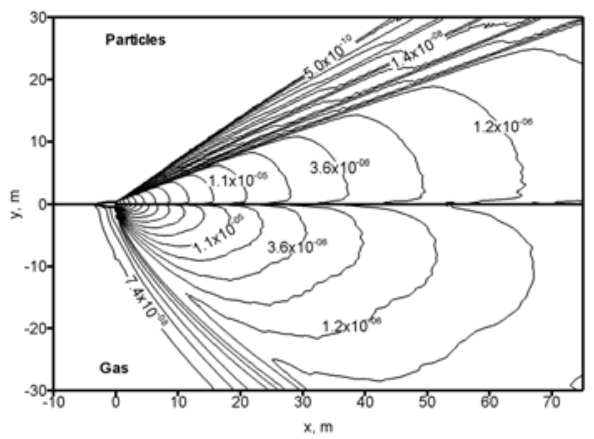

Fig. 7 Contours of mass density for particle and gas. Values are shown in $\mathrm{kg} / \mathrm{m}^{3}$.

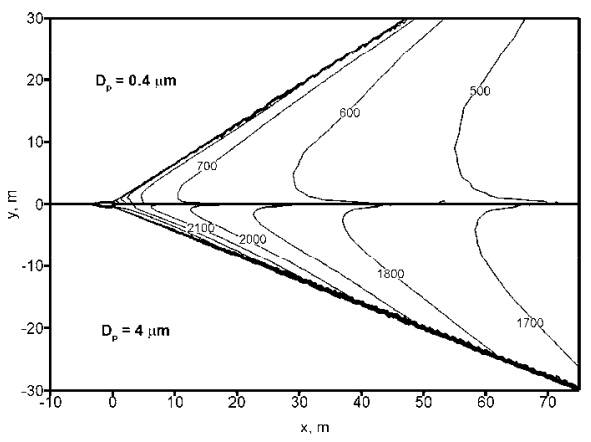

Fig. 9 Average temperature contours for 0.4 and $4 \mu \mathrm{m}$ diameter particles. Values are in $\mathrm{K}$.

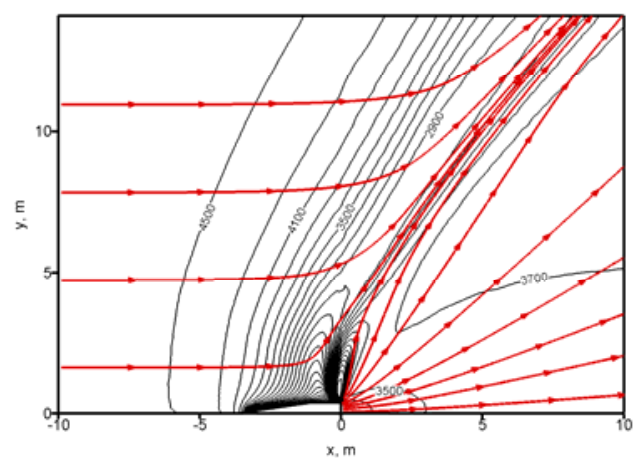

Fig. 6 Gas streamlines and contours of bulk velocity magnitude. Contour line values are given in $\mathbf{m} / \mathbf{s}$.

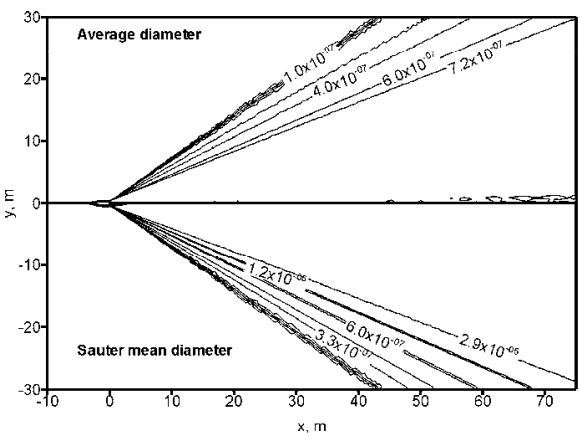

Fig. 8 Contours of average particle diameter and Sauter mean diameter. Values are in $\mathbf{m}$.

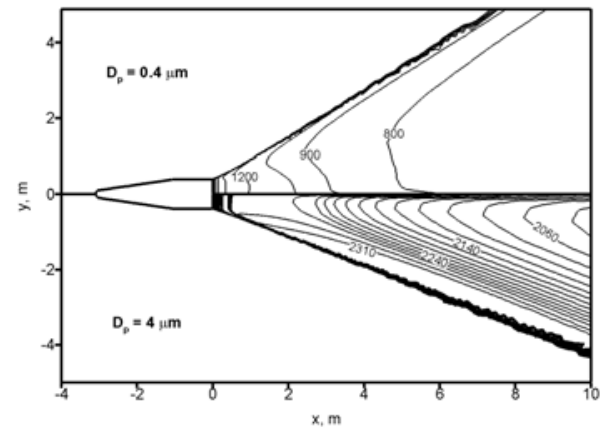

Fig. 10 Close-up view of particle temperature contours. Values are in $K$. 


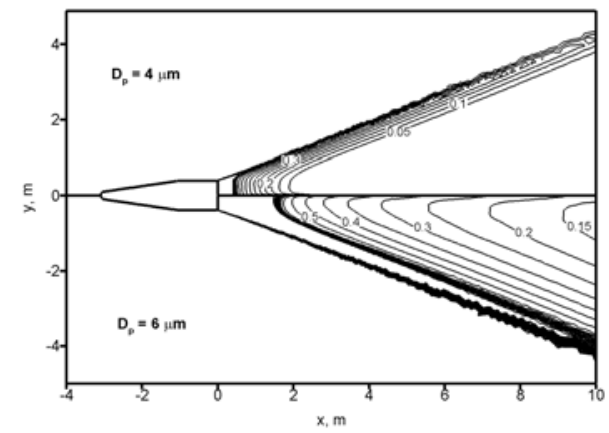

Fig. 11 Contours of liquid mass fraction for 4 and $6 \mu \mathrm{m}$ diameter particles.

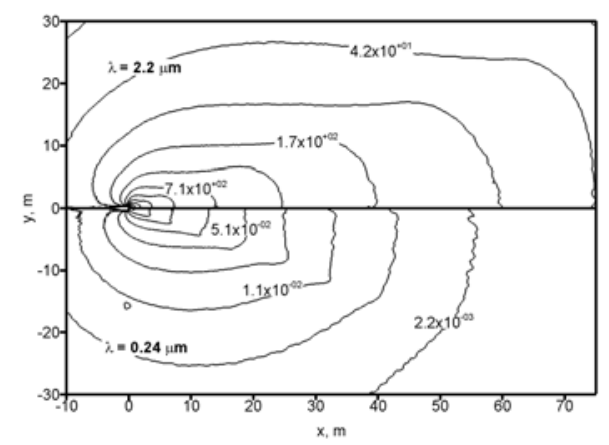

Fig. 13 Contours of the direction-averaged spectral energy flux at wavelengths of $2.2 \mu \mathrm{m}$ and $0.24 \mu \mathrm{m}$. Values are in $\mathrm{W} / \mathrm{m}^{2} \mu \mathrm{m}$.

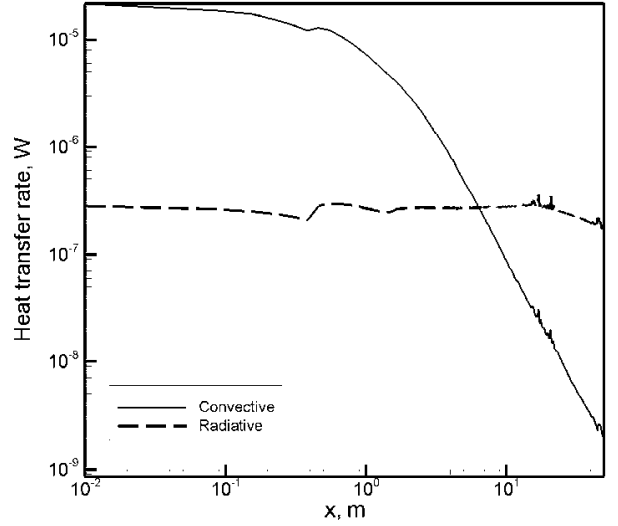

Fig. 12 Magnitude of average convective and radiative heat transfer rates per particle along the centerline.

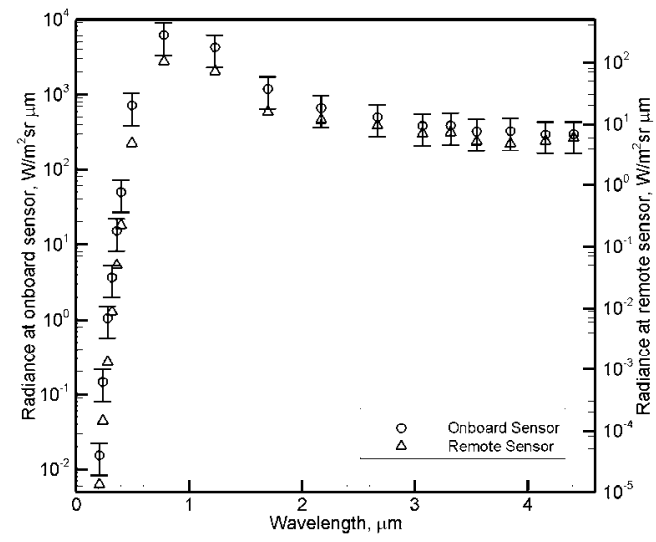

Fig. 14 Spectral radiance at onboard and remote sensors.

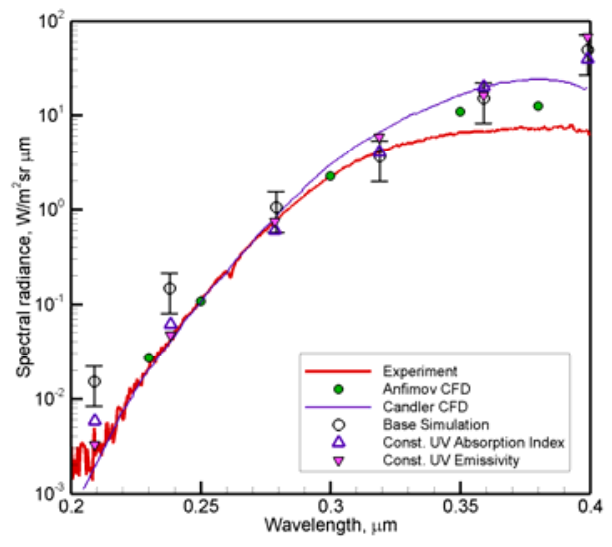

Fig. 15 UV spectral radiance measured at the onboard sensor. 